\title{
Integrative analysis of miRNA-mRNA network in idiopathic membranous nephropathy by bioinformatics analysis
}

\author{
Wenfang He ${ }^{\text {Equal first author, 1, } 2}$, Jinshi Zhang Equal first author, 2 , Shizhu Yuan ${ }^{2}$, Mingzhu Liang ${ }^{2}$, Weidong Chen ${ }^{\text {Corresp., } 1}$, Juan Jin \\ Corresp. 3 \\ 1 Department of Nephrology, The First Affiliated Hospital of Bengbu Medical College, Anhui, China \\ 2 Department of Nephrology, Zhejiang Provincial People's Hospital, Hangzhou, China \\ 3 Department of Nephrology, Affiliated Linan People's Hospital $\square$ Hangzhou Medical College, Hangzhou, China \\ Corresponding Authors: Weidong Chen, Juan Jin \\ Email address: cwd2012@163.com, lang_018@163.com
}

Background.Currently, Several specific antigens, M-type receptor for secretory phospholipase A2(PLA2R1), thrombospondin type-1 domain-containing 7A(THSD7A), and neural epidermal growth factor-like 1 protein (NELL-1), are discovered associated with the onset of idiopathic membranous nephropathy(IMN). But the pathomechanisms of IMNarestill needed to be further claried. Understanding the mechanisms of IMN is required to improve its diagnosis and treatment. Methods. In this study, we constructed miRNA regulatory networks to investigate IMN development. Moreover, miRNAs and mRNAs that were differentially expressed between Idiopathic Membranous Nephropathy(IMN) patients and normal controls were examined using the GSE115857 dataset and our previous sequence study. DE miRNA target genes were determined based on the FUNRICH software, starBase, miRDB, and miRWalk, and an miRNA-mRNA network was designed using DEmRNAs that were negatively correlated with DE-miRNAs. The miRNA-mRNA network contained 228 miRNA-mRNA pairs. Thereafter, we conducted KEGG pathway, GO functional annotation, immune-related gene screening, protein interaction networks, and potential hub gene analyses. Furthermore, 10 miRNAs and 10 genes were determined and preliminarily validated using the validation dataset from GEO. Finally, we identified which pair may offer more accurate diagnosis and therapeutic targets for IMN. Results. two miRNA-mRNA pairs, miR-155-5p-FOS and miR-146a-5p-BTG2, were differentially expressed in IMN, indicating that these genes may affect IMN through immune processes. These findings may offer more accurate diagnoses and therapeutic targets for IMN. 


\section{Integrative Analysis of miRNA-mRNA Network in 2 Idiopathic Membranous Nephropathy by 3 Bioinformatics Analysis}

4

5 Wenfang $\mathrm{He}^{1}$, 2*, Jinshi Zhang ${ }^{2 *}$, Shizhu Yuan², Mingzhu Liang ${ }^{2}$, Weidong Chen ${ }^{1 \#}$, Juan Jin ${ }^{3 \#}$

6 Wenfang He and Jinshi Zhang contributed equally to this work.

$7{ }^{1}$ Department of Nephrology, The First Affiliated Hospital of Bengbu Medical College, Bengbu, 8 Anhui, P.R. China.

92 Department of Nephrology, Zhejiang Provincial People's Hospital, Zhejiang 310014, P.R. 10 China

$11{ }^{3}$ Department of Nephrology, Affiliated Linan People's Hospital, Hangzhou Medical College, 12 Zhejiang 310014, P.R. China.

13

14

Corresponding Author:

Weidong Chen\#: Department of Nephrology, The First Affiliated Hospital of Bengbu Medical College, Anhui0552, P.R. China.

Email address: cwd2012@163.com

Juan Jin": Department of Nephrology, Affiliated Linan People's Hospital, Hangzhou Medical College, Zhejiang 310014, P.R. China.

Email address: lang_018@163.com

\section{Abstract}

Background. Specific antigens such as the M-type receptor for secretory phospholipase A2(PLA2R1), thrombospondin type-1 domain-containing 7A(THSD7A), and neural epidermal growth factor-like 1 protein (NELL-1) are discovered associated with the onset of idiopathic membranous nephropathy (IMN). However, the pathomechanisms underlying the development of IMN are unclear. Understanding the mechanisms of IMN is required to improve its diagnosis and treatment.

Methods. In this study, we constructed miRNA regulatory networks to investigate IMN development. Moreover, miRNAs and mRNAs that were differentially expressed between Idiopathic Membranous Nephropathy(IMN) patients and normal controls were examined using the GSE1 15857 dataset and our previous sequence study. DE miRNA target genes were determined based on the FUNRICH software, starBase, miRDB, and miRWalk, and an miRNAmRNA network was designed using DE-mRNAs that were negatively correlated with DEmiRNAs. The miRNA-mRNA network contained 228 miRNA-mRNA pairs. Thereafter, we conducted KEGG pathway, GO functional annotation, immune-related gene screening, protein interaction networks, and potential hub gene analyses. Furthermore, 10 miRNAs and 10 genes 
were determined and preliminarily validated using the validation dataset from GEO. Finally, we identified which pair may offer more accurate diagnosis and therapeutic targets for IMN.

Results. two miRNA-mRNA pairs, miR-155-5p-FOS and miR-146a-5p-BTG2, were differentially expressed in IMN, indicating that these genes may affect IMN through immune processes. These findings may offer more accurate diagnoses and therapeutic targets for IMN.

Key words Idiopathic Membranous Nephropathy, Bioinformatics Analysis, miRNA-mRNA

\section{Introduction}

Studies have shown that idiopathic membranous nephropathy (IMN) is a prevalent type of nephrotic syndrome among adults (Ponticelli \& Glassock 2014) and is associated with increased prevalence, particularly in China (Xu et al. 2016). It is the second leading cause of end-stage renal disease (ESRD) in patients with primary glomerulonephritis (Ronco \& Debiec 2015). IMN is characterised by subepithelial deposits and diffusely thickened glomerular basement membranes, with clinical presentation of severe proteinuria, oedema, hypoalbuminemia, and hyperlipidaemia (Hull \& Goldsmith 2008). Membranous nephropathy (MN) is an organ-specific autoimmune disease. Podocytes are regarded as the primary targets of the immunological response, which often involves podocyte antigens(Ronco \& Debiec 2020). A few auto-antigens have been identified in adult IMN including PLA2R1, THSD7A, and NELL-1(Liu et al. 2020). The understanding of the pathogenesis and diagnoses, as well as treatment, has improved because of the identification of these new indicators.

Podocyte maintenance may play an important role in the prevention and treatment of membranous nephropathy and may indirectly improve its damage by blocking the input of immune stress(Iranzad et al. 2021). Reducing the immune response to prevent the development of IMN by regulating immune-related genes may serve as an important breakthrough. However, several factors in IMN still require further study and clarification. Besides, given our limited understanding of the detailed mechanism of immune-associated genesin IMN development, treatment with costly and potentially toxic drugs is challenging. Therefore, exploration of IMN mechanisms is required to improve its diagnosis and treatment.

MicroRNAs are non-coding RNAs that are 21-24 nucleotides in length. MicroRNAs drive disease pathogenesis by regulating expression levels of their specific target genes (Esteller 2011). Published studies have shown that miRNA dysregulation contributes to IMN development, and is therefore a potential drug target for IMN treatment (Barbagallo et al. 2019; Li et al. 2019; Sun et al. 2020). In our previous study, the expression patterns of urinary exosomal miRNAs varied between IMN samples and healthy control samples (Zhang et al. 2020). Thus, exploring the relationships between miRNAs and target genes in IMN may assist in early diagnosis and treatment. 
In this study, we aimed to integrate the analysis of IMN-related miRNAs and mRNAs, acquired through the GSE1 15857 dataset from GEO and our previous sequence study (Zhang et al. 2020). A miRNA-mRNA regulatory network was established from DE-mRNAs that were negatively correlated with DE-miRNAs. Thereafter, KEGG pathway analysis, GO functional annotation, immune-related gene screening, PPI network analysis, and potential hub gene analyses were also conducted. Finally, potential hub genes and miRNAs were selected and preliminarily validated using the GSE133288 and GSE64306 datasets from GEO. The present study may help elucidate the pathogenic mechanism to improve the diagnosis, prognosis, and therapy of IMN.

\section{Materials \& Methods}

\section{Data source}

The miRNA expression profile obtained from our previous study, which included urinary exosomal miRNAs, contained five healthy controls and six subjects with IMN (Zhang et al. 2020). mRNA expression profiles were downloaded from the GEO database. The main inclusion criteria were as follows: (1) the dataset included kidney tissue from IMN patients and healthy volunteers and (2) the samples in each group did not undergo any specific treatment. After rational screening, GSE1 15857 was included in the present study. After rational screening, GSE1 15857, GSE133288, and GSE64306 were included in our study. The microarray data from GSE1 15857 were based on the GPL14951 (Illumina HumanHT-12) platform and contained seven healthy controls and 11 patients with IMN. GSE133288 comprised mRNA data and included 48 patients with IMN and five healthy controls, involving the probe platform GPL19983 (Affymetrix Human Gene 2.1 ST Array). GSE64306 comprised miRNA data and included three patients with IMN and six healthy controls whose probe platform was GPL19117 (Affymetrix Multispecies miRNA-4 Array).

\section{Acquisition of differential expression miRNAs and mRNAs}

Differentially expressed mRNAs (DE-mRNAs) between IMN and non-IMN volunteer samples of GSE1 15857 were identified using the limma package in R (version 3.36.3) (Gentleman et al. 2004) with thresholds of $|\log \mathrm{FC}|>1$ and adjusted $P$-values $<0.05$. Differentially expressed miRNAs (DE-miRNAs) between the IMN and non-IMN volunteer samples were assessed using the EdgeR package (edgeR 3.14.0) (Robinson et al. 2010) in R. Significant DE-miRNAs were selected using $|\operatorname{logFC}|>1$ and adjusted $P$-values $<0.05$.

\section{The prediction of target genes of DE-miRNA and establishment of a DE miRNA-DE mRNA regulatory network}

Target mRNAs of DE-miRNAs were predicted using three prediction tools: FUNRICH software (Version 3.1.3), miRWalk (http://mirwalk.umm.uni-heidelberg.de), and miRDB (http://mirdb.org/). The overlapping genes between targets and DE-mRNAs were selected, and the DE-mRNAs that negatively correlated with DE-miRNAs were further screened. The 
118 miRNA-mRNA regulatory network was created using the Cytoscape software (Smoot et al.

119 2011).

120

121

122

Determination of pathways and biological processes linked to DE-mRNAs

123

The KEGG pathway and GO enrichment assessments were performed on DE-mRNAs in the

124 regulatory network using Enrichr (https://maayanlab.cloud/Enrichr) (Kuleshov et al. 2016). The cut-off value was set at $P<0.05$.

125

126

\section{Immune-related DE-mRNAs}

127

Immune-related genes were identified based on the InnateDB database. InnateDB is a manually

128 curated knowledge base of genes involved in human innate immunity (Breuer et al. 2013). Thus, we screened DE-mRNAs in the regulatory network against immune-associated genes in the InnateDB database. Overlapped genes were considered MN-related immune DE-mRNAs and were used in the following analysis.

132

\section{PPI Networks of MN-related immune DE-mRNAs}

134

The PPI network of DE-mRNAs was created using the STRING database (http://string-db.org/)

(Szklarczyk et al. 2011), a tool for analysing functional interactions between DE-mRNAs. Hub

genes were identified by calculating the CytoHubba plugin from the Cytoscape software (Smoot

et al. 2011). Hub gene scores of connectivity were calculated using the Maximal Clique

138 Centrality algorithm (MCC), which is described as:

$$
\operatorname{MCC}(v)=\sum_{C \in S(v)}(|C|-1) !, \text { where } S(v) \text { is }
$$

the collection of maximal cliques which contain $v$, and $(|C|-1)$ ! is the product of positive integers less than $|\mathrm{C}|($ Chin et al. 2014).

\section{Results}

\section{Identification of DE mRNAs and miRNAs} limma package in R 3.5.3 (adjusted $P$-value $<0.05$, $|\log \mathrm{FC}|>1$ ) (Fig. 1A, B). According to the analysis of our previous study (Zhang et al. 2020), a total of 849 miRNAs were differentially expressed between the IMN and CON groups, 43 miRNAs were significantly upregulated, and 15 miRNAs were significantly downregulated according to the criteria of adjusted $P$-value $<$ $0.05,|\log \mathrm{FC}|>1$ (Fig. 1C, D).

\section{Establishment of a miRNA-mRNA network}

152 The DE miRNA target genes were predicted using the prediction tools described above, including the FUNRICH software, miRDB, and miRWalk. The predicted target genes 
155

156

157

158

159

160

161

162

163

164

165

166

167

168

169

170

171

172

173

174

175

176

177

178

179

180

181

182

183

184

185

186

187

188

189

190

191

192

193

194

corresponding DE miRNA targets were selected as key DE mRNAs (Fig.S1). Finally, we obtained 139 key DE mRNAs, and designed a miRNA-mRNA regulatory network (Fig 2A), which included 228 miRNA-mRNA pairs. The cytoHubba plugin selected the top 10 nodes with the highest score of connectivity, and 10 miRNAs were defined as hub miRNAs (Fig2B).

\section{Functional annotation analysis of key genes}

The GO enrichment for 139 key genes in the regulatory network showed that the most enriched terms were "negative regulation of cellular process," "mitotic nuclear envelope reassembly," and "regulation of transcription from RNA polymerase II promoter" in the BP category; "catenin complex," "specific granule membrane," and "ionotropic glutamate receptor complex" in the CC category; and "core promoter proximal region sequence-specific DNA binding" and "RNA polymerase II core promoter proximal region sequence-specific DNA binding" in the MF category (Fig.S2). Furthermore, findings from KEGG analyses suggested that the genes were predominantly linked to the MAPK signalling pathway, osteoclast differentiation, B cell receptor signalling pathway, viral carcinogenesis, and IMN-associated pathways (Fig. S3).

\section{Screened immune-related genes}

In further analyses, we explored the innate immune genes that were altered in IMN using the InnateDB database. A total of 59 IMN-related immune-associated genes were included in further analysis (Fig. 3A).

\section{PPI network of the IMN-related immune-associated genes}

Next, IMN-related immune-associated genes in the PPI networks were explored to determine the hub genes. The PPI network comprised 47 edges and 59 nodes (Fig. 3B). The CytoHubba plugin in Cytoscape was used to identify hub genes, and tencandidates were selected (Fig. 3C).

\section{Identification of hub gene and miRNA expression using validation dataset}

We demonstrated the hub miRNA using the GSE64306 dataset. We focused on the expression levels of hsa-miR-155-5p, hsa-miR-4516, hsa-miR-1269a, hsa-miR-4492, hsa-miR-197-3p, hsamiR-9-5p, hsa-miR-146a-5p, hsa-miR-23b-5p, hsa-miR-194-3p, and hsa-miR-204-3p. As a result, we found that hsa-miR-155-5p, hsa-miR-146a-5p, and hsa-miR-4492 showed significant expression changes in the validation dataset (Fig. 4). Another mRNA expression profile dataset GSE133288 from GEO was used for hub mRNA validation. We investigated the expression levels of FOS, JUN, FOSL1, BTG2, NR4A1, MAFB, MAFG, HIST2H2BE, and IL1A. We observed that FOS, JUN, BTG2, and NR4A1 showed significant differential expression in the validation dataset (Fig. 5). Comparing the two results, we observed that two miRNA-mRNA pairs, miR-155-5p-FOS and miR-146a-5p-BTG2, were differentially expressed in IMN.

\section{Discussion}

Numerous studies have shown that immune and epigenetic processes influence the development 
195

196

197

198

199

200

201

202

203

204

205

206

207

208

209

210

211

212

213

214

215

216

217

218

219

220

221

222

223

224

225

226

227

228

229

230

231

232

233

of IMN (Roccatello et al. 2016; Sha et al. 2015). Among epigenetic disruptions, changes in miRNA levels may alter immune functions and hence affect the prognosis of IMN. Understanding the impact of abnormal miRNA expression in IMN will help in developing strategies for diagnosing and treating IMN (Barbagallo et al. 2019; Motavalli et al. 2021).

Previous studies have shown that urinary exosomes contain miRNAs originating from the tubular and glomerular cells (Salih et al. 2014). Here, we comprehensively assessed the differential expression levels of miRNA in urinary exosomes and mRNA in the kidney tissues of IMN patients, 43 DE miRNAs and 815 DE mRNAs. We further predicted the DE miRNA target genes and overlapped them with DE mRNAs, and then constructed the miRNA-mRNA regulatory network, which was composed of 139 key genes and 228 miRNA-mRNA pairs. For the 139 key genes in the regulatory network, three GO_BP terms (such as negative regulation of cellular process), three GO_CC terms (such as catenin complex), and three GO_MF terms (such as RNA polymerase II core promoter proximal region sequence-specific DNA binding) were enriched.

\section{Results from KEGG pathway analyses demonstrated that several important genes were enriched} in the MAPK, B cell receptor, and osteoclast differentiation pathways. Among these pathways, the B cell receptor pathway is known to influence the B cell signalling network and stimulate autoimmune disease pathogenesis, resulting in IMN (Motavalli et al. 2019). The MAPK signalling pathway also plays a significant role in the immunomodulatory effects of $\mathrm{T}$ cells and B cells (Dennison et al. 2021). The results of functional annotation analysis suggest that immune-related genes warrant future investigation.

Therefore, we screened 139 key genes that correlated with immunity using the InnateDB database. A total of 59 immune-associated genes were screened to construct a PPI network. The CytoHubba plugin of Cytoscape was used to analyse the miRNA-mRNA regulatory network and the immune-associated gene PPI network. Tenhub genes and 10 hub miRNAs were screened. Next, the hub miRNAs and genes were identified by analysing these against the validation dataset. Finally, two miRNA-mRNA pairs, miR-155-5p-FOS and miR-146a-5p-BTG2, were found to affect immune processes and offer more accurate diagnosis and therapeutic targets for IMN.

MiR-155-5p is a multifunctional miRNA which is overexpressed in the kidney tubules of diabetic kidney and renal fibrosis patients (Baker et al. 2017). Recent research has revealed that miR-155-5p can cause oxidative stress and inflammation in diabetic kidneys and renal fibrosis by attenuating the autophagic process (Guo et al. 2020; Wang et al. 2020; Wang et al. 2018). Our previous study showed that autophagy inhibition aids IMN-induced podocyte injury (Jin et al. 2018). In summary, miR-155-5p may be essential for the regulation of the autophagic process in podocytes of IMN.

Peer] reviewing PDF | (2021:05:61388:2:0:NEW 7 Sep 2021) 
234 Genes in FOS encode leucine zipper proteins, which are involved in the dimerisation of proteins 235 belonging to the JUN family. Thus, they lead to the formation of transcription factor activator 236 protein (AP-1). FOS proteins modulate differentiation, proliferation, and apoptotic cell death 237 (Tang et al. 2020). Previous investigations have shown that the $F O S$ gene is also involved in 238 immune system regulation (Wagner \& Eferl 2005). Recent research has revealed that FOS can 239 affect the transcription of inflammatory cytokines by binding to their promoters, leading to their 240 high expression inducing endotoxin-induced and cisplatin-induced acute kidney injury (AKI)

241 (Miyazaki et al. 2012; Zhang et al. 2019). There is considerable evidence that inflammatory 242 processes contribute to the pathogenesis of IMN (Nangaku et al. 2005; Wu et al. 2008). These 243 results confirmed the association between FOS and IMN.

244

245

246

247

248

249

250

251

252

253

254

255

256

257

258

259

260

261

262

263

264

265

266

267

268

269

270

271

272

273

MiRNA-146a-5p was first reported in humans in 2006 (Taganov et al. 2006) and is a well-known miRNA associated with inflammation (Rusca \& Monticelli 2011). It was revealed that miR-146a expression is upregulated in the kidney, where it induces chronic inflammation (Ichii et al.

2012). The effects of miR-146a-5p have been demonstrated in renal diseases and can be used for the treatment of renal fibrosis and ischemia/reperfusion injury by inhibiting the infiltration of inflammatory cells ( $\mathrm{Li}$ et al. 2020; Morishita et al. 2015). From this, we can speculate that miRNA-146a-5p may influence IMN through its effects on inflammation.

BTG2 belongs to the B cell translocation gene in the BTG/Tob family, which is associated with several cellular processes (Mauxion et al. 2009; Winkler 2010; Yuniati et al. 2019). It is noteworthy that BTG2 was highly expressed in lymph nodes and white blood cells, suggesting its role in the immune system. BTG1 deficiency leads to inappropriate T-cell clonal expansion, while differentiation can cause immune-related diseases (Hwang et al. 2020). Consistent with the literature, our research found that the expression of BTG2 was abnormally low in patients with IMN.

There are some limitations in our study. The datasets GSE115857, GSE133288, and GSE64306 were obtained from the GEO database and were generated by different researchers, this may result in certain biases during gene analysis. Further, as shown in some similar studies, we only showed the opposites interacting of miRNAs and mRNA. miRNA-mRNA regulatory network in IMN were based on target gene prediction and statistical evidence, further experimental validation is required to verify the results.

\section{Conclusions}

We present a miRNA-mRNA regulatory network consisting of 228 miRNA-mRNA pairs in IMN. We found that two miRNA-mRNA pairs, miR-155-5p-FOS and miR-146a-5p-BTG2, were differentially expressed in IMN, indicating that these genes may affect IMN through immune processes. These findings may offer more accurate diagnoses and therapeutic targets for IMN. Further experimental validation is required to verify the results.

Peer) reviewing PDF | (2021:05:61388:2:0:NEW 7 Sep 2021) 
274

275

276

277

278

279

280

281

282

283

284

285

286

287

288

289

290

291

292

293

294

295

296

297

298

299

300

301

302

303

304

305

306

307

308

309

310

311

312

313

314

315

316

317

\section{Acknowledgements}

We would like to thank Editage (www.editage.cn) for English language editing.

\section{Funding}

This research was supported by the Construction of Key Projects by Zhejiang Provincial

Ministry (Grant Number: WKJ-ZJ-1915),the General Project of Zhejiang Education

Department (Grant Number: Y201942823),Clinical and Experimental Research of YSHS

Granule.

\section{Availability of data and materials}

The expression data associated with this article is available on GEO databases

(https://www.ncbi.nlm.nih.gov/geo/).

\section{Authors' contributions}

Wenfang He and Jinshi Zhang performed the comparative analysis using bioinformatics tools. Shizhu Yuan and Mingzhu Liang participated in the data analysis and discussion. Wenfang He and Jinshi Zhang interpreted the results and wrote the manuscript. Juan Jin and Weidong Chen organized and supervised the project. All authors read and approved the final manuscript.

Wenfang He and Jinshi Zhang contributed equally to this work.

\section{Competing interests}

No potential conflict of interest was reported by the author.

\section{References}

Baker M, Davis S, Liu P, Pan X, Williams A, Iczkowski K, Gallagher S, Bishop K, Regner K, Liu Y, and Liang M. 2017. Tissue-Specific MicroRNA Expression Patterns in Four Types of Kidney Disease. Journal of the American Society of Nephrology : JASN 28:2985-2992. 10.1681/asn.2016121280

Barbagallo C, Passanisi R, Mirabella F, Cirnigliaro M, Costanzo A, Lauretta G, Barbagallo D, Bianchi C, Pagni F, Castorina S, Granata A, Di Pietro C, Ragusa M, Malatino L, and Purrello M. 2019. Upregulated microRNAs in membranous glomerulonephropathy are associated with significant downregulation of IL6 and MYC mRNAs. Journal of cellular physiology 234:1262512636. 10.1002/jcp.27851

Breuer K, Foroushani A, Laird M, Chen C, Sribnaia A, Lo R, Winsor G, Hancock R, Brinkman F, and Lynn D. 2013. InnateDB: systems biology of innate immunity and beyond--recent updates and continuing curation. Nucleic acids research 41:D1228-1233. 10.1093/nar/gks1147

Chin $\mathrm{CH}$, Chen SH, Wu HH, Ho CW, Ko MT, and Lin CY. 2014. cytoHubba: identifying hub objects and sub-networks from complex interactome. BMC Syst Biol 8 Suppl 4:S11. 10.1186/1752-0509-8-s4-s11

Dennison L, Mohan A, and Yarchoan M. 2021. Tumor and Systemic Immunomodulatory Effects of MEK Inhibition. Current oncology reports 23:23. 10.1007/s11912-020-01008-4

Esteller M. 2011. Non-coding RNAs in human disease. Nature reviews Genetics 12:861-874. 10.1038/nrg3074

Gentleman R, Carey V, Bates D, Bolstad B, Dettling M, Dudoit S, Ellis B, Gautier L, Ge Y, Gentry J, Hornik K, Hothorn T, Huber W, lacus S, Irizarry R, Leisch F, Li C, Maechler M, Rossini A, Sawitzki G, Smith C, Smyth G, Tierney L, Yang J, and Zhang J. 2004. 
318

319

320

321

322

323

324

325

326

327

328

329

330

331

332

333

334

335

336

337

338

339

340

341

342

343

344

345

346

347

348

349

350

351

352

353

354

355

356

357

358

359

360

361

362

363

364

365

366

367

368
Bioconductor: open software development for computational biology and bioinformatics. Genome biology 5:R80. 10.1186/gb-2004-5-10-r80

Guo L, Tan K, Luo Q, and Bai X. 2020. Dihydromyricetin promotes autophagy and attenuates renal interstitial fibrosis by regulating miR-155-5p/PTEN signaling in diabetic nephropathy. Bosnian journal of basic medical sciences 20:372-380. 10.17305/bjbms.2019.4410

Hull R, and Goldsmith D. 2008. Nephrotic syndrome in adults. BMJ (Clinical research ed) 336:1185-1189. 10.1136/bmj.39576.709711.80

Hwang S, Lim J, Yu Z, Kong P, Sefik E, Xu H, Harman C, Kim L, Lee G, Li H, and Flavell R. 2020. mRNA destabilization by BTG1 and BTG2 maintains T cell quiescence. Science (New York, NY) 367:1255-1260. 10.1126/science.aax0194

Ichii O, Otsuka S, Sasaki N, Namiki Y, Hashimoto Y, and Kon Y. 2012. Altered expression of microRNA miR-146a correlates with the development of chronic renal inflammation. Kidney international 81:280-292. 10.1038/ki.2011.345

Iranzad R, Motavalli R, Ghassabi A, Pourakbari R, Etemadi J, and Yousefi M. 2021. Roles of microRNAs in renal disorders related to primary podocyte dysfunction. Life Sci 277:119463. 10.1016/j.Ifs.2021.119463

Jin J, Hu K, Ye M, Wu D, and He Q. 2018. Rapamycin Reduces Podocyte Apoptosis and is Involved in Autophagy and mTOR/ P70S6K/4EBP1 Signaling. Cellular physiology and biochemistry : international journal of experimental cellular physiology, biochemistry, and pharmacology 48:765-772. 10.1159/000491905

Kuleshov M, Jones M, Rouillard A, Fernandez N, Duan Q, Wang Z, Koplev S, Jenkins S, Jagodnik K, Lachmann A, McDermott M, Monteiro C, Gundersen G, and Ma'ayan A. 2016. Enrichr: a comprehensive gene set enrichment analysis web server 2016 update. Nucleic acids research 44:W90-97. 10.1093/nar/gkw377

Li J, Chen Y, Shen L, and Deng Y. 2019. Improvement of membranous nephropathy by inhibition of miR-193a to affect podocytosis via targeting WT1. Journal of cellular biochemistry 120:3438-3446. 10.1002/jcb.27616

Li X, Liao J, Su X, Li W, Bi Z, Wang J, Su Q, Huang H, Wei Y, Gao Y, Li J, Liu L, and Wang C. 2020. miR-146a-5pHuman urine-derived stem cells protect against renal ischemia/reperfusion injury in a rat model via exosomal which targets. Theranostics 10:95619578. 10.7150/thno.42153

Liu W, Gao C, Liu Z, Dai H, Feng Z, Dong Z, Zheng Y, Gao Y, Tian X, and Liu B. 2020. Idiopathic Membranous Nephropathy: Glomerular Pathological Pattern Caused by Extrarenal Immunity Activity. Front Immunol 11:1846. 10.3389/fimmu.2020.01846

Mauxion F, Chen C, Séraphin B, and Shyu A. 2009. BTG/TOB factors impact deadenylases. Trends in biochemical sciences 34:640-647. 10.1016/j.tibs.2009.07.008

Miyazaki H, Morishita J, Ueki M, Nishina K, Shiozawa S, and Maekawa N. 2012. The effects of a selective inhibitor of c-Fos/activator protein-1 on endotoxin-induced acute kidney injury in mice. BMC nephrology 13:153. 10.1186/1471-2369-13-153

Morishita Y, Imai T, Yoshizawa H, Watanabe M, Ishibashi K, Muto S, and Nagata D. 2015. Delivery of microRNA-146a with polyethylenimine nanoparticles inhibits renal fibrosis in vivo. International journal of nanomedicine 10:3475-3488. 10.2147/ijn.S82587

Motavalli R, Etemadi J, Kahroba H, Mehdizadeh A, and Yousefi M. 2019. Immune systemmediated cellular and molecular mechanisms in idiopathic membranous nephropathy pathogenesis and possible therapeutic targets. Life sciences 238:116923. 10.1016/j.Ifs.2019.116923

Motavalli R, Etemadi J, Soltani-Zangbar M, Ardalan M, Kahroba H, Roshangar L, Nouri M, Aghebati-Maleki L, Khiavi F, Abediazar S, Mehdizadeh A, Hojjat-Farsangi M, Mahmoodpoor A, Kafil H, Zolfaghari M, Ahmadian Heris J, and Yousefi M. 2021. Altered Th17/Treg ratio as a possible mechanism in pathogenesis of idiopathic membranous nephropathy. Cytokine 141:155452. 10.1016/j.cyto.2021.155452 
369

370

371

372

373

374

375

376

377

378

379

380

381

382

383

384

385

386

387

388

389

390

391

392

393

394

395

396

397

398

399

400

401

402

403

404

405

406

407

408

409

410

411

412

413

414

415

416

417
Nangaku M, Shankland S, and Couser W. 2005. Cellular response to injury in membranous nephropathy. Journal of the American Society of Nephrology : JASN 16:1195-1204. 10.1681/asn.2004121098

Ponticelli C, and Glassock R. 2014. Glomerular diseases: membranous nephropathy--a modern view. Clinical journal of the American Society of Nephrology : CJASN 9:609-616. $10.2215 /$ cjn. 04160413

Robinson M, McCarthy D, and Smyth G. 2010. edgeR: a Bioconductor package for differential expression analysis of digital gene expression data. Bioinformatics (Oxford, England) 26:139140. 10.1093/bioinformatics/btp616

Roccatello D, Sciascia S, Di Simone D, Solfietti L, Naretto C, Fenoglio R, Baldovino S, and Menegatti E. 2016. New insights into immune mechanisms underlying response to Rituximab in patients with membranous nephropathy: A prospective study and a review of the literature. Autoimmunity reviews 15:529-538. 10.1016/j.autrev.2016.02.014

Ronco P, and Debiec H. 2015. Pathophysiological advances in membranous nephropathy: time for a shift in patient's care. Lancet (London, England) 385:1983-1992. 10.1016/s01406736(15)60731-0

Ronco P, and Debiec H. 2020. Molecular Pathogenesis of Membranous Nephropathy. Annu Rev Pathol 15:287-313. 10.1146/annurev-pathol-020117-043811

Rusca N, and Monticelli S. 2011. MiR-146a in Immunity and Disease. Molecular biology international 2011:437301. 10.4061/2011/437301

Salih M, Zietse R, and Hoorn E. 2014. Urinary extracellular vesicles and the kidney: biomarkers and beyond. American journal of physiology Renal physiology 306:F1251-1259. 10.1152/ajprenal.00128.2014

Sha W, Shen L, Zhou L, Xu D, and Lu G. 2015. Down-regulation of miR-186 contributes to podocytes apoptosis in membranous nephropathy. Biomedicine \& pharmacotherapy $=$ Biomedecine \& pharmacotherapie 75:179-184. 10.1016/j.biopha.2015.07.021

Smoot M, Ono K, Ruscheinski J, Wang P, and Ideker T. 2011. Cytoscape 2.8: new features for data integration and network visualization. Bioinformatics (Oxford, England) 27:431-432. 10.1093/bioinformatics/btq675

Sun Z, Xu Q, Ma Y, Yang S, and Shi J. 2020. Circ_0000524/miR-500a-5p/CXCL16 axis promotes podocyte apoptosis in membranous nephropathy. European journal of clinical investigation:e13414. 10.1111/eci.13414

Szklarczyk D, Franceschini A, Kuhn M, Simonovic M, Roth A, Minguez P, Doerks T, Stark M, Muller J, Bork P, Jensen L, and von Mering C. 2011. The STRING database in 2011: functional interaction networks of proteins, globally integrated and scored. Nucleic acids research 39:D561-568. 10.1093/nar/gkq973

Taganov K, Boldin M, Chang K, and Baltimore D. 2006. NF-kappaB-dependent induction of microRNA miR-146, an inhibitor targeted to signaling proteins of innate immune responses. Proceedings of the National Academy of Sciences of the United States of America 103:12481-12486. 10.1073/pnas.0605298103

Tang H, Tang Y, Zeng T, and Chen L. 2020. Gene expression analysis reveals the tipping points during infant brain development for human and chimpanzee. BMC genomics 21:74. 10.1186/s12864-020-6465-8

Wagner E, and Eferl R. 2005. Fos/AP-1 proteins in bone and the immune system. Immunological reviews 208:126-140. 10.1111/j.0105-2896.2005.00332.x

Wang G, Wu B, Zhang B, Wang K, and Wang H. 2020. LncRNA CTBP1-AS2 alleviates high glucose-induced oxidative stress, ECM accumulation, and inflammation in diabetic nephropathy via miR-155-5p/FOXO1 axis. Biochemical and biophysical research communications 532:308-314. 10.1016/j.bbrc.2020.08.073 
418

419

420

421

422

423

424

425

426

427

428

429

430

431

432

433

434

435

436

437

438

439

440

441

442

443
Wang Y, Zheng Z, Jia Y, Yang Y, and Xue Y. 2018. Role of p53/miR-155-5p/sirt1 loop in renal tubular injury of diabetic kidney disease. Journal of translational medicine 16:146. 10.1186/s12967-018-1486-7

Winkler G. 2010. The mammalian anti-proliferative BTG/Tob protein family. Journal of cellular physiology 222:66-72. 10.1002/jcp.21919

Wu C, Lu K, Chen J, Hsieh H, Lin S, Chu P, Wang J, Sytwu H, and Lin Y. 2008. HO-1 induction ameliorates experimental murine membranous nephropathy: anti-oxidative, anti-apoptotic and immunomodulatory effects. Nephrology, dialysis, transplantation : official publication of the European Dialysis and Transplant Association - European Renal Association 23:30823090. 10.1093/ndt/gfn247

Xu X, Wang G, Chen N, Lu T, Nie S, Xu G, Zhang P, Luo Y, Wang Y, Wang X, Schwartz J, Geng J, and Hou F. 2016. Long-Term Exposure to Air Pollution and Increased Risk of Membranous Nephropathy in China. Journal of the American Society of Nephrology : JASN 27:3739-3746. 10.1681/asn.2016010093

Yuniati L, Scheijen B, van der Meer L, and van Leeuwen F. 2019. Tumor suppressors BTG1 and BTG2: Beyond growth control. Journal of cellular physiology 234:5379-5389. 10.1002/jcp.27407

Zhang C, Ma P, Zhao Z, Jiang N, Lian D, Huo P, and Yang H. 2019. miRNA-mRNA regulatory network analysis of mesenchymal stem cell treatment in cisplatin-induced acute kidney injury identifies roles for miR-210/Serpine1 and miR-378/Fos in regulating inflammation. Molecular medicine reports 20:1509-1522. 10.3892/mmr.2019.10383

Zhang J, Zhu Y, Cai R, Jin J, and He Q. 2020. Differential Expression of Urinary Exosomal Small RNAs in Idiopathic Membranous Nephropathy. BioMed research international 2020:3170927. 10.1155/2020/3170927 
Figure 1

Profiling of DE mRNAs and DE miRNAs in IMN.

(A) Heat map and (B) Volcano plot of DE mRNAs among IMN samples and healthy controls.

(C) Heat map and (D) Volcano plot of DE miRNAs among IMN patients and healthy controls.

Red represents upregulation and blue represents downregulation.

A

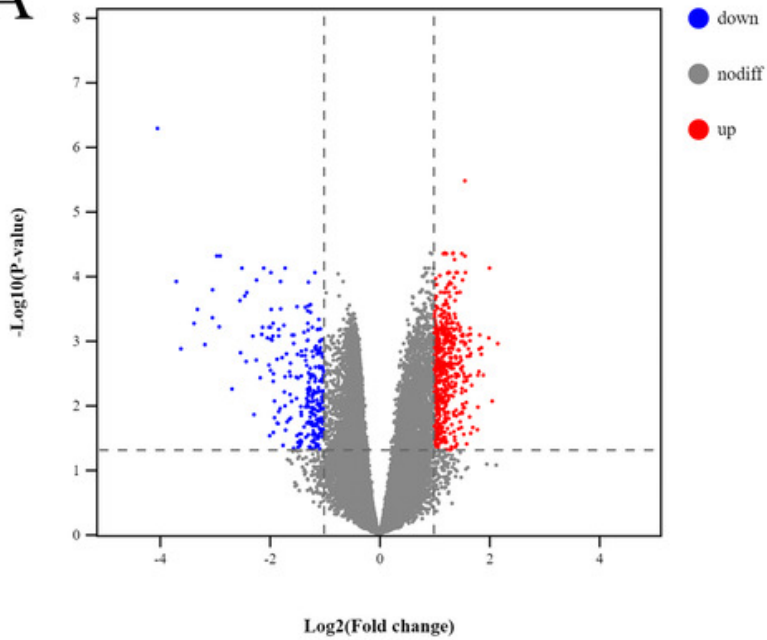

$\mathrm{C}$

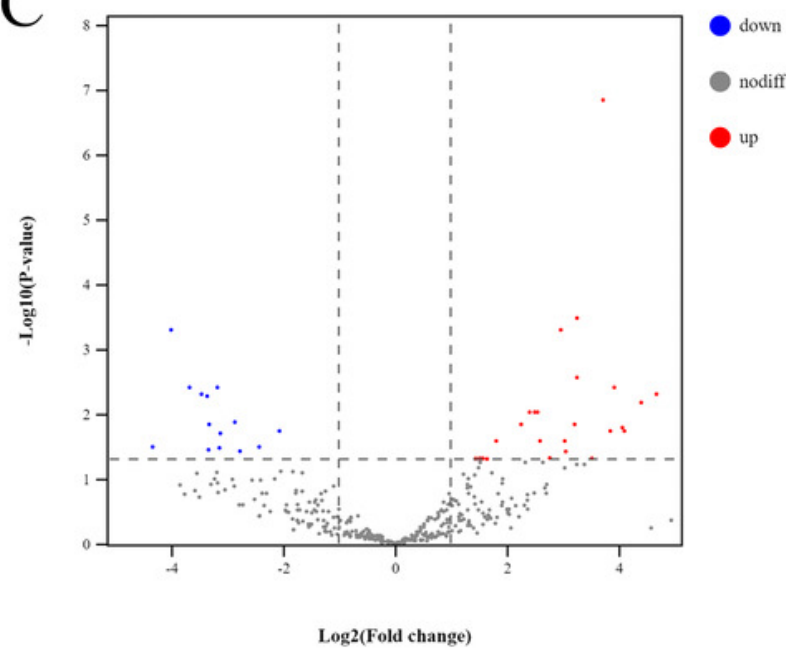

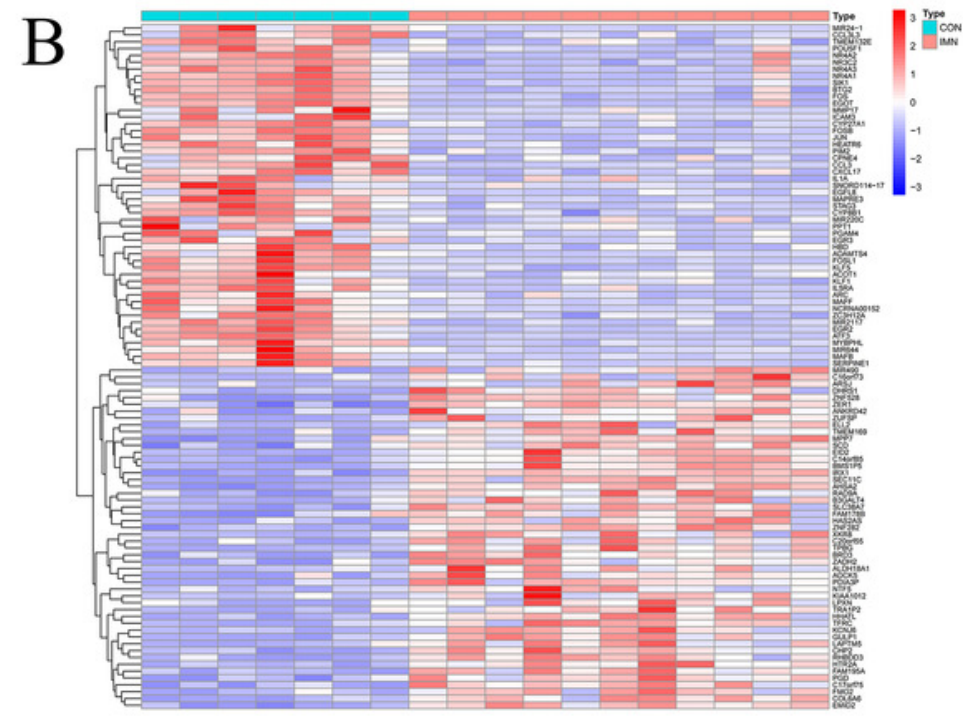

$\mathrm{D}$

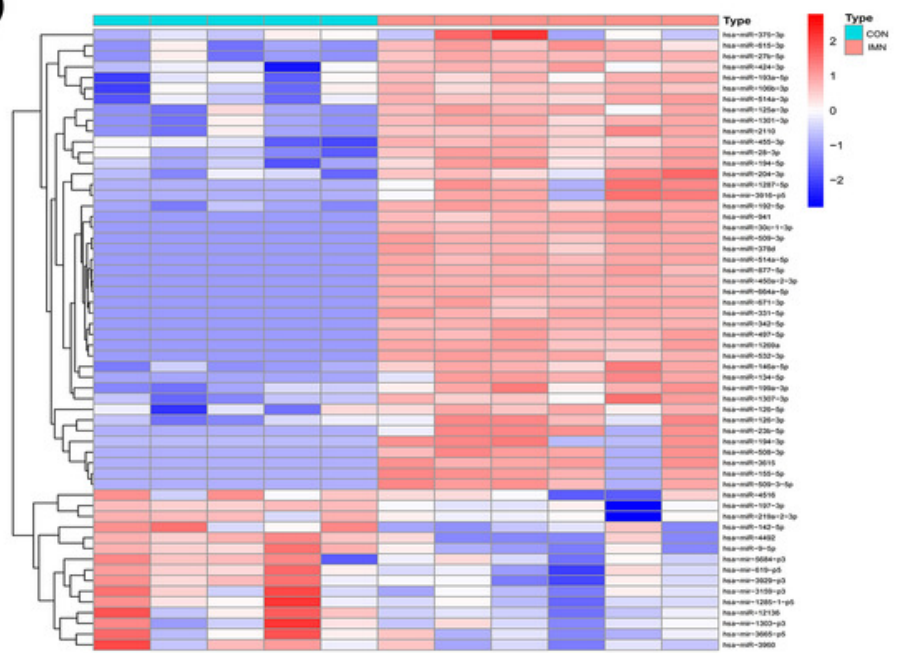


Figure 2

The miRNA-mRNA regulatory network in IMN and interaction between 10 hub miRNAs and target DE mRNAs.

(A) The miRNA-mRNA regulatory network in IMN. The triangles represent DE-miRNAs, and the ellipses represent target DE-mRNAs. Red indicates upregulation and blue indicates downregulation. (B) The interaction between 10 hub miRNAs and target DE mRNAs.

A

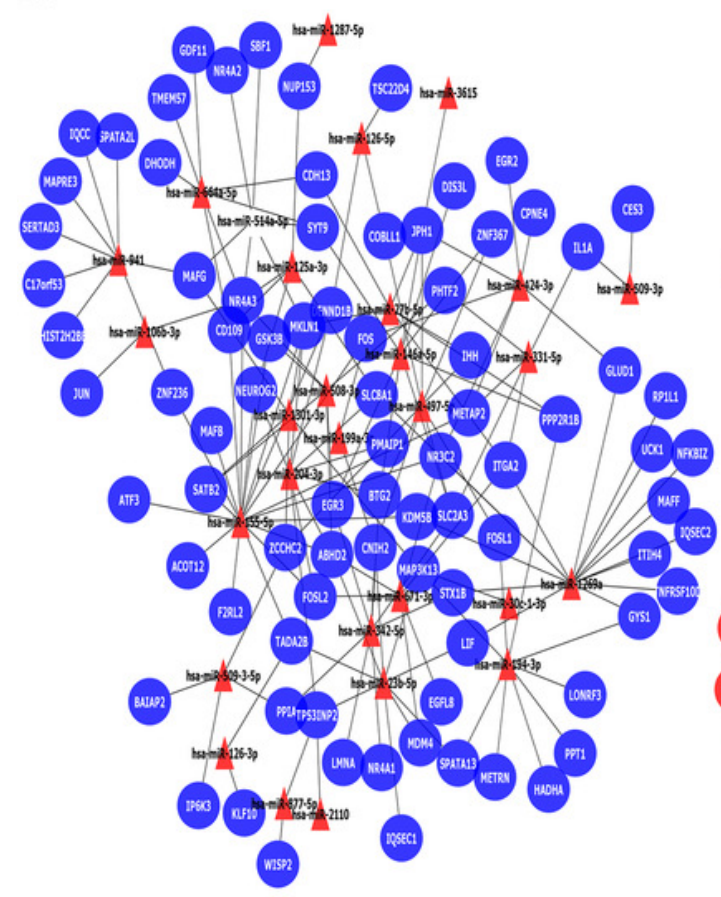

B

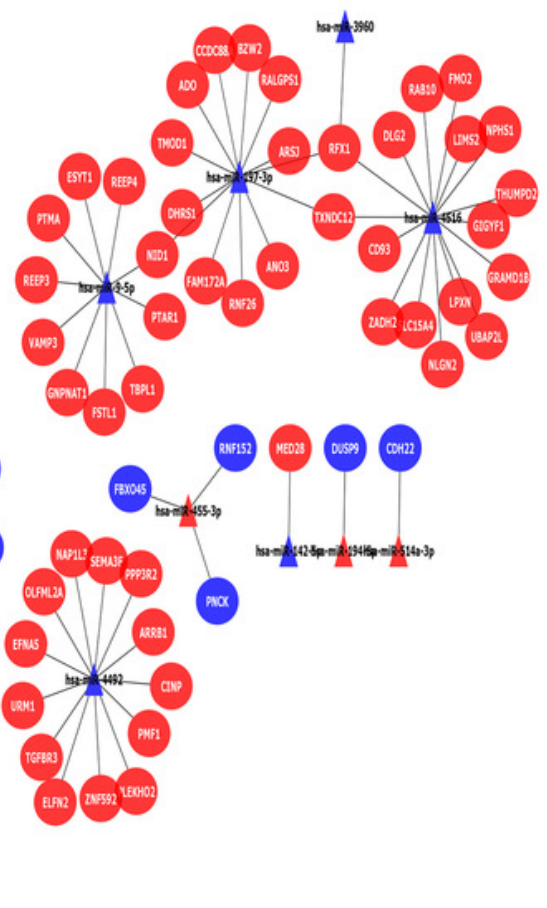

\begin{tabular}{|ccc|}
\hline Rank & Name & Score \\
\hline 1 & hss-miR-155-5p & 19 \\
\hline 2 & hss-miR-4516 & 16 \\
\hline 3 & hss-miR-1269a & 15 \\
\hline 4 & hss-miR-4492 & 13 \\
\hline 4 & hss-miR-197-3p & 13 \\
\hline 6 & hsa-miR-9.5p & 10 \\
\hline 7 & hsa-miR-1466-5p & 9 \\
\hline 7 & hsa-miR-23b-5p & 9 \\
\hline 7 & hss-miR-194-3p & 9 \\
\hline 7 & hsa-miR-204-3p & 9 \\
\hline
\end{tabular}




\section{Figure 3}

The miRNA-mRNA regulatory network in IMN and top ten miRNAs with the highest score of interaction in the miRNA-mRNA regulatory network.

(A) The miRNA-mRNA regulatory network in IMN. The triangles represent DE-miRNAs, and the ellipses represent target DE-mRNAs. Red indicates upregulation and blue indicates downregulation. (B) The interaction between 10 hub miRNAs and target DE mRNAs. (C) Top ten miRNAs with the highest score of interaction in the miRNA-mRNA regulatory network.

A

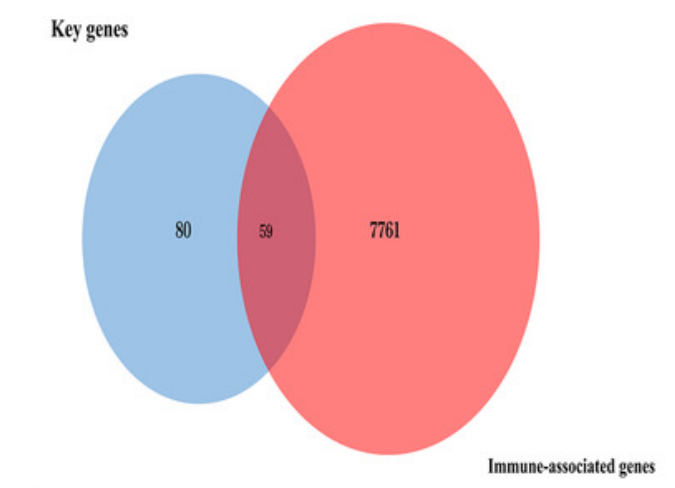

C

\begin{tabular}{|ccc|}
\hline Rank & Name & Score \\
\hline 1 & FOS & 49 \\
\hline 2 & JUN & 46 \\
\hline 3 & FOSL1 & 37 \\
\hline 4 & BTG2 & 24 \\
\hline 4 & NR4A1 & 24 \\
\hline 6 & MAFB & 12 \\
\hline 7 & MAFG & 6 \\
\hline 7 & HIST2H2BE & 6 \\
\hline 9 & IL1A & 5 \\
\hline 10 & ARRB1 & 4 \\
\hline
\end{tabular}

B

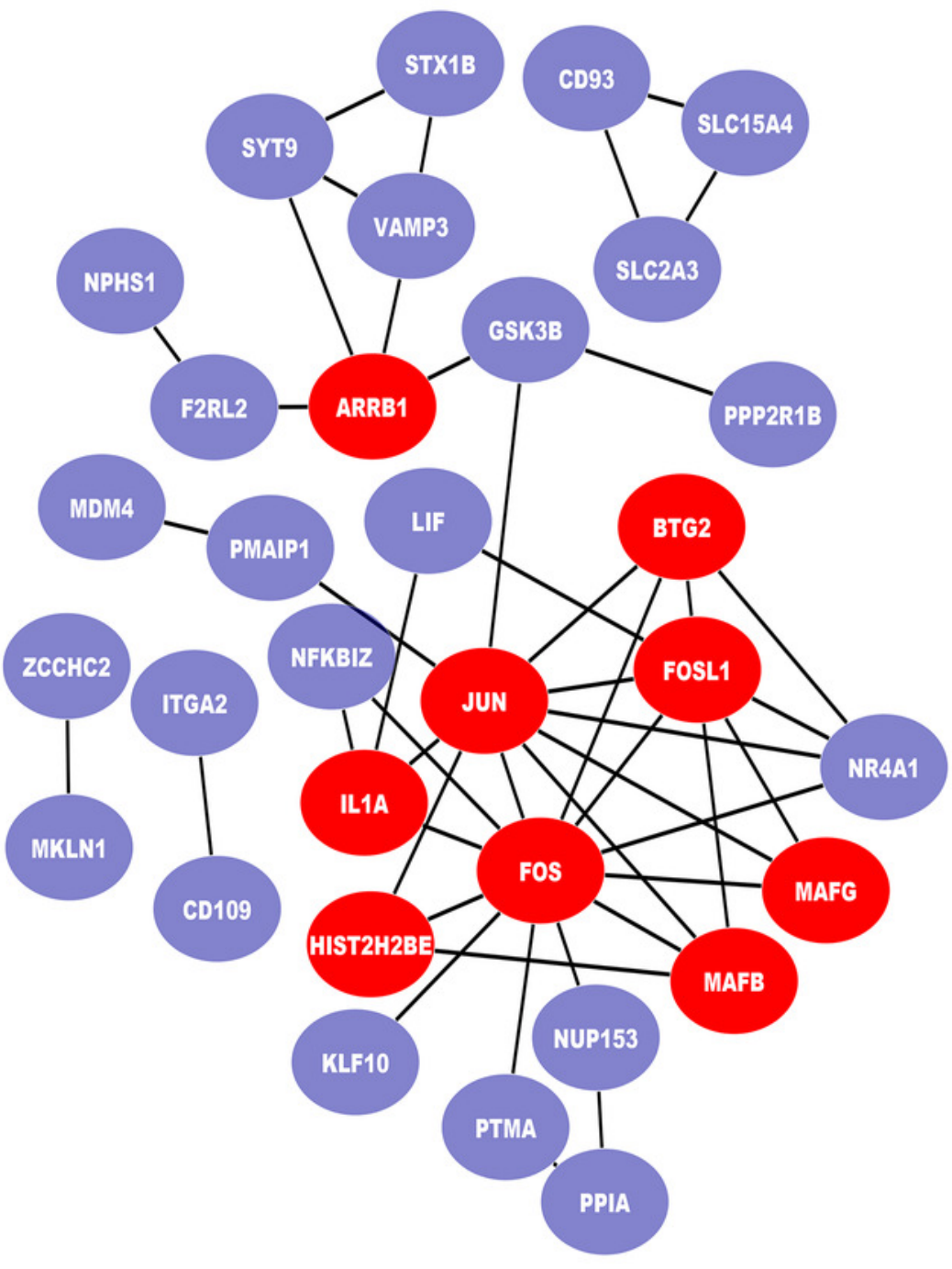


Figure 4

Expression profile of hub miRNAs in the validation database (GSE64306).
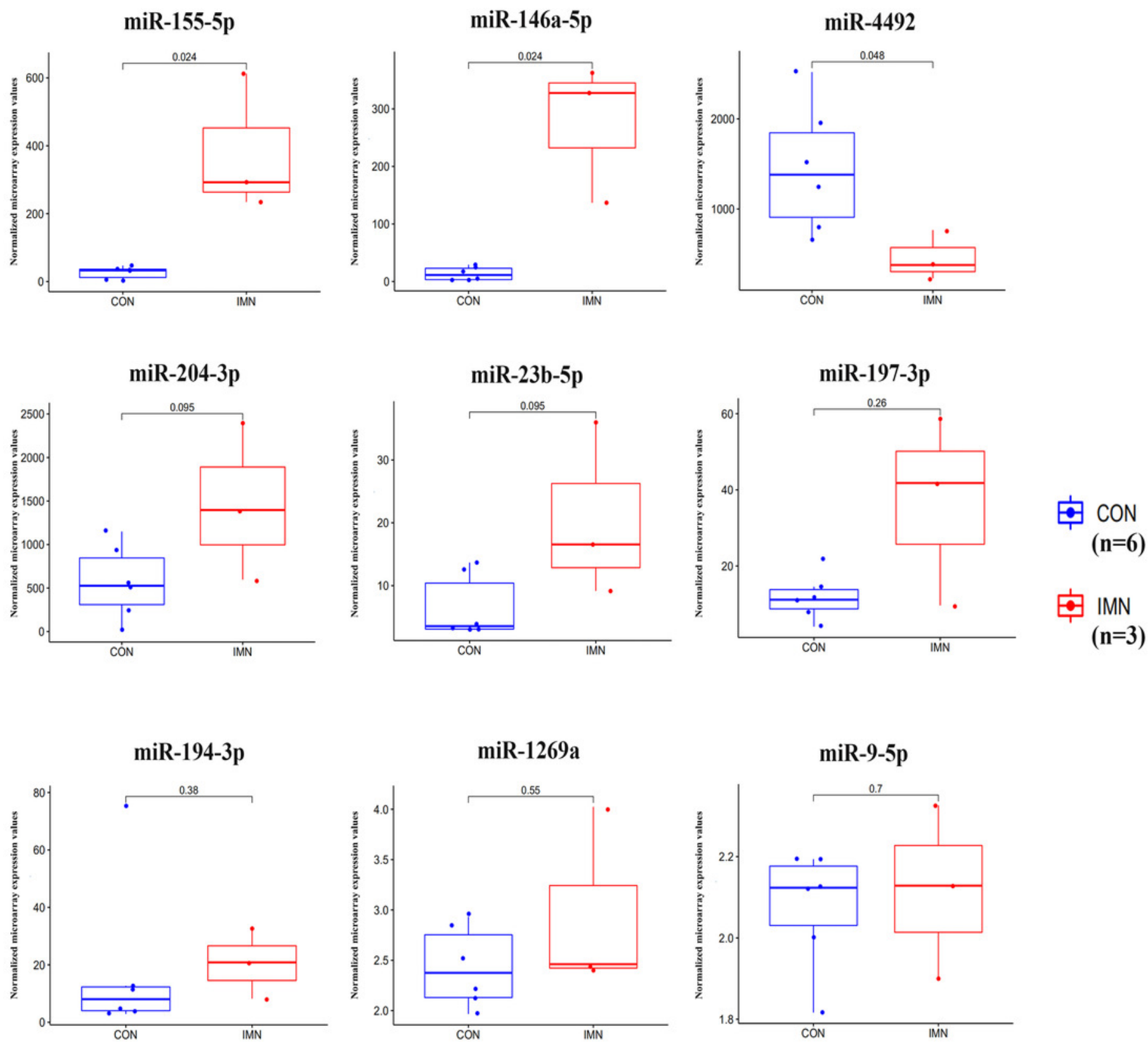
Figure 5

Expression profile of hub genes in the validation database (GSE133288).
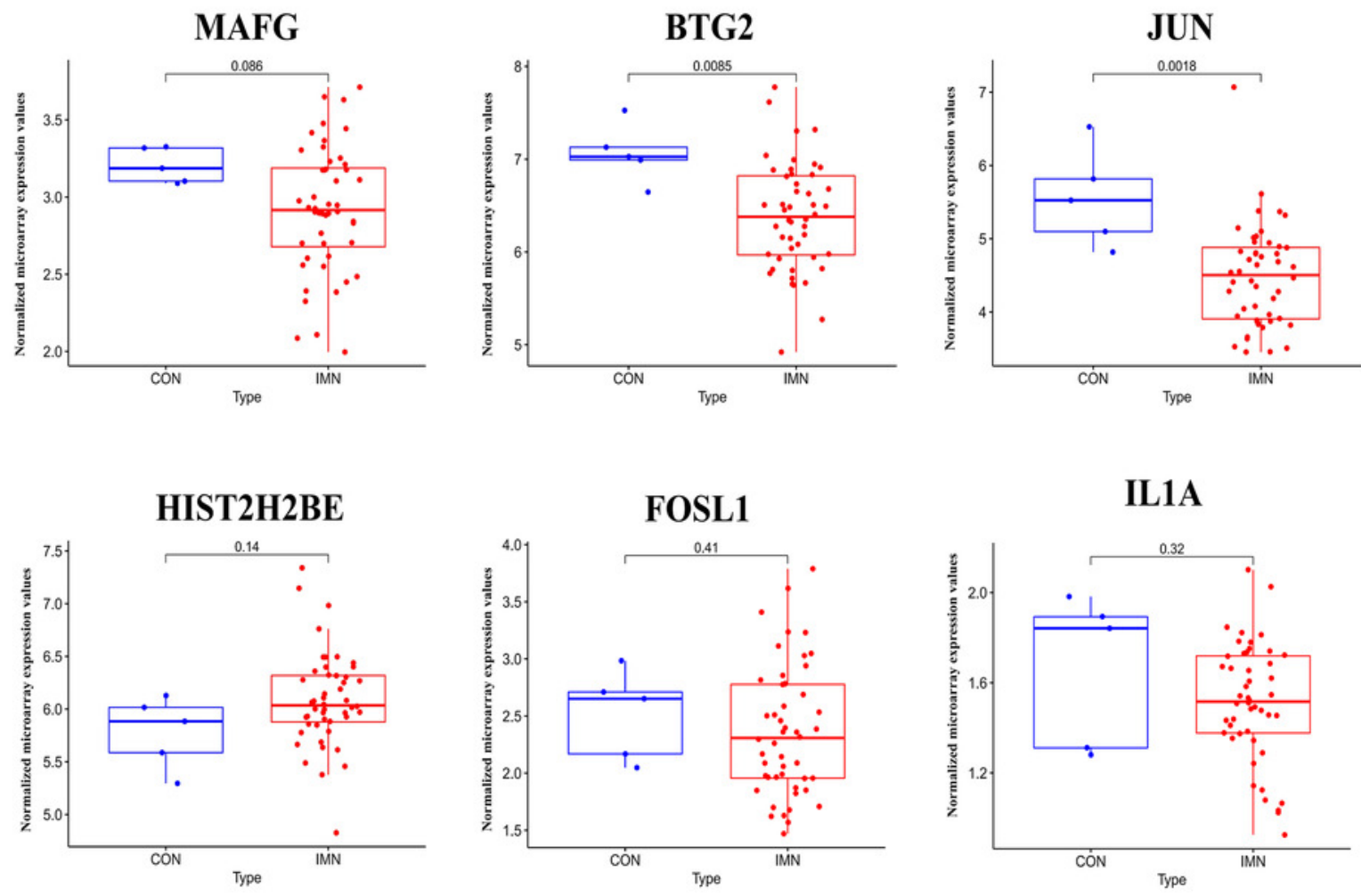

安 CON

$(\mathrm{N}=5)$

审 IMN

$(\mathrm{N}=48)$
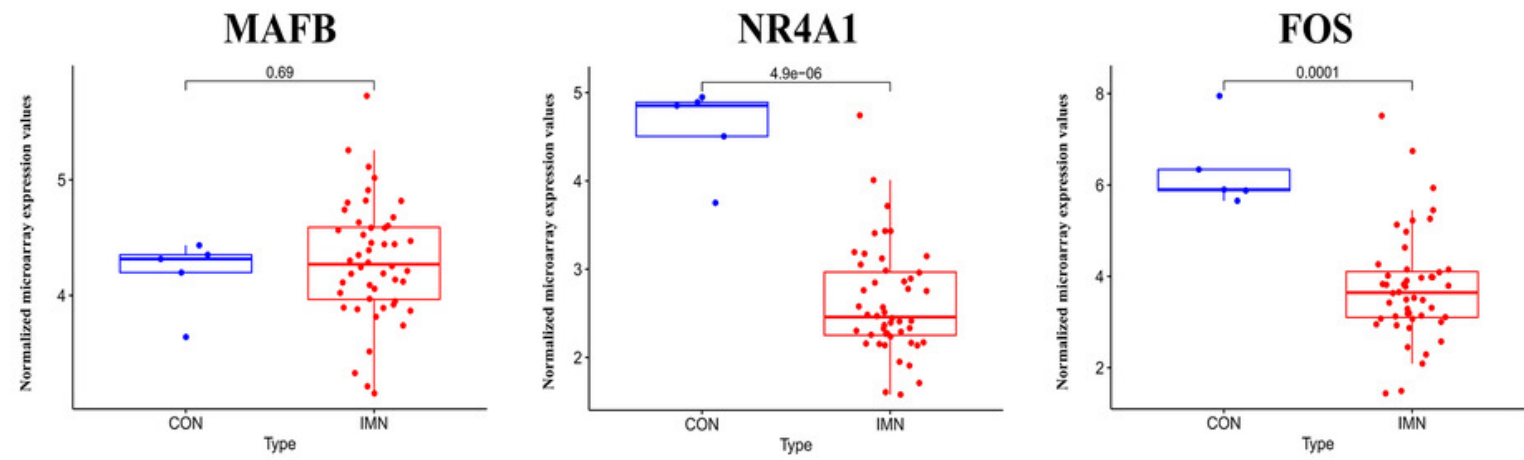